\title{
Far-Field Autofluorescence Nanoscopy
}

\author{
Jakob Bierwagen, ${ }^{\dagger, \S}$ Ilaria Testa, ${ }^{\dagger, \S}$ Jonas Fölling, ${ }^{\dagger, \S}$ Dirk Wenzel, ${ }^{\ddagger}$ Stefan Jakobs, ${ }^{\dagger}$ \\ Christian Eggeling, ${ }^{\dagger}$ and Stefan W. Hell ${ }^{*}{ }^{\dagger}$
}

\author{
$\dagger^{\dagger}$ Max Planck Institute for Biophysical Chemistry, Department of NanoBiophotonics and ₹Electron Microscopy \\ Facility, Am Fassberg 11, 37077 Göttingen, Germany
}

\begin{abstract}
We demonstrate far-field optical imaging at the nanoscale with unlabeled samples. Subdiffraction resolution images of autofluorescent samples are obtained by depleting the ground state of natural fluorophores by transferring them to a metastable dark state and simultaneously localizing those fluorophores that are transiently returning. Our approach is based on the insight that nanoscopy methods relying on stochastic single-molecule switching require only a single fluorescence on-off cycle to yield an image, a condition fulfilled by various biomolecules. The method is exemplified by recording label-free nanoscopy images of thylakoid membranes of spinach chloroplasts.
\end{abstract}

KEYWORDS Microscopy, resolution, chlorophyll, ground state depletion, nanoscopy, GSDIM

M any substances in biological tissue display natural autofluorescence, examples being aromatic amino acids, lipo-pigments, NADH, NADPH, FADH, collagene, elastin, flavins, carotenoids, or chlorophyll. ${ }^{1}$ In fluorescence microscopy, autofluorescence is perturbing since the signal is superimposed with the signal from specifically labeled parts of the sample. However, if the cellular structures of interest themselves are scattering ${ }^{2}$ or fluorescent, they can be observed without additional staining. Autofluorescence far-field imaging has been employed in diagnostics and microscopy, but in all of these implementations, the imaging resolution was limited by diffraction to about half the wavelength of light (>200 nm).

In recent years, several far-field optical microscopy methods emerged for overcoming the diffraction limit. ${ }^{3-15}$ Therefore, the question arises as to whether some of them operate with autofluorescence. This question is particularly interesting because all methods rely on transferring closely spaced fluorophores between distinct states to make the fluorophores distinguishable, ${ }^{3,4,7,15}$ with the most straightforward pair of states being a fluorescent "on" and a nonfluorescent

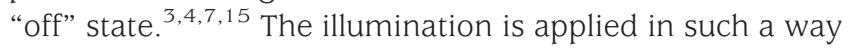
that not all but only a subset of fluorophores is able to reside in the "on" state at the time point of detection. For example, in stimulated emission depletion (STED) ${ }^{3,5}$ and ground state depletion (GSD) ${ }^{4,10}$ nanoscopy fluorophores are only able to dwell in the fluorescent on state, when they are in the subdiffractional proximity of an intensity minimum of the STED or GSD beam. The other fluorophores residing within diffraction-limited distances ( $\sim 200 \mathrm{~nm}$ ) are transiently kept dark by STED or GSD, respectively. Although they are exposed to the excitation light, these fluorophores are

\footnotetext{
* To whom correspondence should be addressed, shell@gwdg.de.

$\S$ I.T., J.B., and J.F. are equal contributors.

Received for review: 08/5/2010

Published on Web: 09/10/2010
}

effectively toggled to a dark off state, which is the ground state in the case of STED or a metastable dark state in the case of GSD. Scanning of the intensity minima across the sample produces the subdiffraction resolution image by sequentially allowing the other fluorophores to occupy the on state.

Whereas in STED, GSD, and related ${ }^{6,8,9}$ nanoscopy methods the location of the on and off state fluorophores is predefined by spatially modulated light (i.e., targeted switching and read-out ${ }^{15,16}$ ), more recent methods switch single fluorophores randomly in space (stochastic switching). ${ }^{11-14}$ Within a region defined by the diffraction limit, roughly a single molecule is stochastically switched to the state; again the other molecules remain dark, although being exposed to excitation light. The train of photons emitted by a fluorophore in the on state is recorded by a camera and the position of the fluorophore determined with subdiffraction accuracy by localization. ${ }^{17,18}$ Afterward, the fluorophore is turned off and another one is stochastically switched on and off to perform the same cycle. Registering sufficient fluorophores in this way yields a subdiffraction image. Known as PALM,${ }^{11}$ STORM,${ }^{12}$ FPALM,${ }^{13}$ and PALMIRA, ${ }^{14}$ initial implementations of this stochastic concept relied on photoactivatable markers such as photoactivatable fluorescent proteins or photochromic organic markers. The on states differed from the off states by molecular conformation or bonds. Therefore, these versions of stochastic optical nanoscopy are unlikely to be viable with autofluorescence, because most natural fluorophores are not photoactivatable. Their resting state is not an off state but an on state; i.e., they can instantly emit fluorescence upon proper illumination.

Since stimulated emission is the most basic transition to reversibly turn "on state" fluorophores off, STED nanoscopy can in principle accommodate autofluorescent samples. However, the normally short lifetimes of the fluorescent states call for rather high STED beam intensities. ${ }^{3,19}$ Nanoscopy by $\mathrm{GSD}^{4,10}$ requires lower intensities as compared to 
STED because of the longer lifetime of the states involved. ${ }^{19}$ However, like STED, GSD nanoscopy employs coordinatetargeted fluorophore switching which requires the fluorophore to undergo many on-off cycles to form an image. ${ }^{15,16}$ This is hard to achieve with autofluorescent molecules because they are prone to bleaching, ${ }^{20,21}$ meaning that they can cycle only a few times between the on and an off state.

An important insight is that stochastic methods can produce an image with just a single on-off cycle per molecule, because in order to be localized on a camera, it is enough for a single molecule to assume the on state just once. ${ }^{15,16}$ Therefore, the most direct pathway to nanoscale imaging of autofluorescent samples is to apply a stochastic switching modality. Thus, in conjunction with a molecular mechanism that produces an off-on-off succession, far-field nanoscopy of autofluorescent samples should become viable.

A switching mechanism that fulfills this condition is GSD, since it can be realized with basically any fluorophore. For this reason, it has also been exploited in the stochastically operating method called GSDIM (ground state depletion with individual molecule return). ${ }^{22-27}$ Contrary to photoactivation, GSD acts on bright, regular fluorophores which are first turned off by transferring them to a metastable dark state, such as the triplet $^{4,10}$ or a redox state. ${ }^{27}$ The transfer occurs via an excited state, usually a fluorescent state, which is why fluorescence is generated during the process (inset Figure 1a).

GSDIM can be conveniently implemented with continuous illumination at a single wavelength ${ }^{22}$ performing both the switching and the fluorescence read out. If the intensity is sufficiently high and the dark state lifetime $\tau$ sufficiently extended, virtually all markers are turned off for a characteristic duration $\tau$. This ensures that ideally just about a single marker remains in the bright on state within a diffraction limited region, so that its position can be localized and registered. Afterward, the marker reenters a dark state, whereas another marker reassumes the bright on state, in a stochastic fashion. ${ }^{22}$

To demonstrate the viability of the concept, we used the autofluorescence of chlorophyll in spinach chloroplasts to obtain subdiffraction resolution GSDIM images of thylakoid membranes in the chloroplasts (Supporting Information). It is known that chlorophyll features several metastable dark states. ${ }^{28,29}$ Transferring most of the chlorophyll into these off states should therefore enable GSDIM. Upon irradiation with excitation light of $488 \mathrm{~nm}$ with up to $100 \mathrm{~kW} / \mathrm{cm}^{2}$ we found that the majority ( $\sim 70 \%$ ) of the fluorescent molecules in the chloroplasts can be turned off (Figure 1a). Most importantly, the fluorescence signal of the molecular ensemble was largely recovered when switching off the excitation light (Figure 1b). The average recovery time, i.e., the average lifetime of the dark states of chlorophyll, $\tau=1.2 \mathrm{~s}$, is about 3-6 orders of magnitude longer than that of typical triplet states in organic dyes in most biological samples.

This transient off-switching of endogenous fluorophores by GSD was applied for nanoscale imaging of chloroplasts.

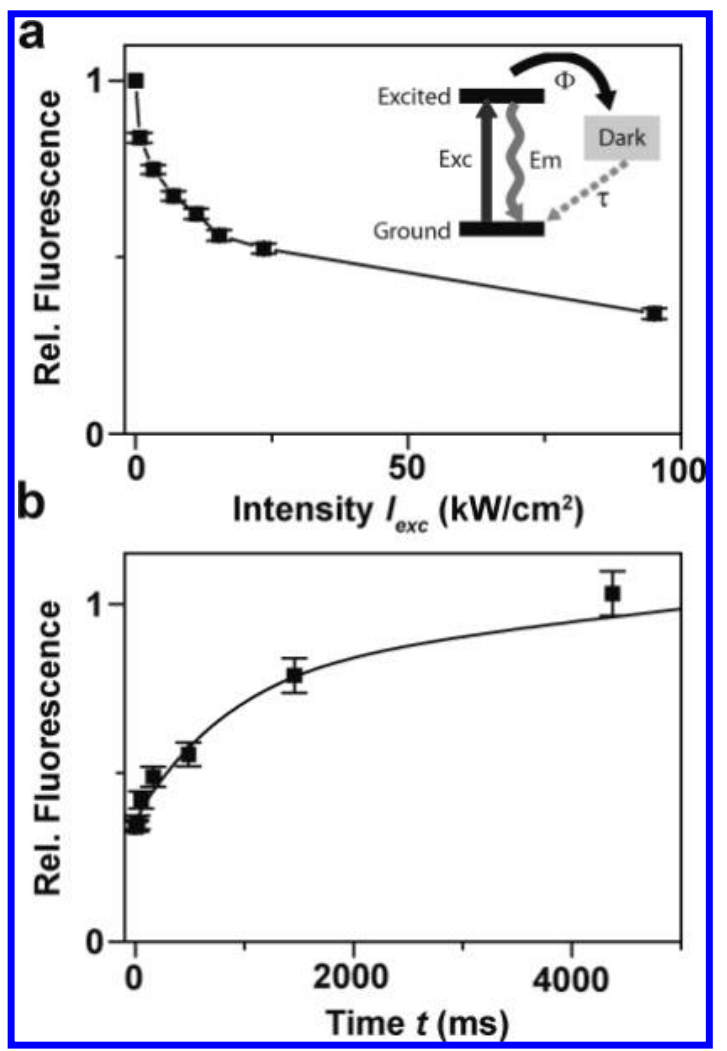

FIGURE 1. Ground state depletion (GSD) of autofluorophores. (a) Depletion of the fluorescent (on) state of chlorophyll with increasing intensity $I_{\text {exc }}$ of the $488 \mathrm{~nm}$ excitation light. The residual fluorescence was read out $\left(100 \mu \mathrm{s}, 1 \mathrm{~kW} / \mathrm{cm}^{2}\right.$ of $488 \mathrm{~nm}$ light) after illumination for $100 \mathrm{~ms}$ with $I_{\mathrm{exc}}$. Inset, GSD is based on an optical transfer into metastable dark states: following optical excitation (Exc) from the ground to the excited (fluorescent, Em) state, crossing to metastable dark states with lifetimes $\tau$ occurs with probability Ф. (b) Recovery of fluorescence of chlorophyll after GSD (black squares). The recovered signal was read out $\left(100 \mu \mathrm{s}, 5 \mathrm{~kW} / \mathrm{cm}^{2}\right.$ of $488 \mathrm{~nm}$ light) at different times $t$ after illumination for $100 \mathrm{~ms}$ with $I_{\mathrm{exc}}=95 \mathrm{~kW} / \mathrm{cm}^{2}$. An exponential fit (solid line) to the data resulted in a characteristic recovery time $\tau=1.2 \mathrm{~s}$.

To reduce the background light from the out-of-focus layers, 75-100 nm thick cryosections of purified chloroplasts were used (Supporting Information). Figure 2a shows a typical electron microscopy image of our chlorophyll samples displaying thylakoid membrane stacks in the chloroplasts. Illuminating an area of approximately $15 \mu \mathrm{m}$ in diameter with $28 \mathrm{~kW} / \mathrm{cm}^{2}$ of $488 \mathrm{~nm}$ laser light turns approximately $50 \%$ of the autofluorophores dark at a time (compare Figure 1a). Due to limited available laser power, higher intensities were not possible in our wide-field microscope, meaning that the fraction of molecules remaining in the on state was still too large for slices of the chosen thickness. The signal of the fluorescing molecules was still overlapping and singlemolecule signatures could not be detected. However, after illumination for $60 \mathrm{~s}$, enough molecules turned dark due to further dark state transitions or photobleaching, so that roughly a single emitter could be detected per diffraction region (Figure $2 \mathrm{~b}$ ). 


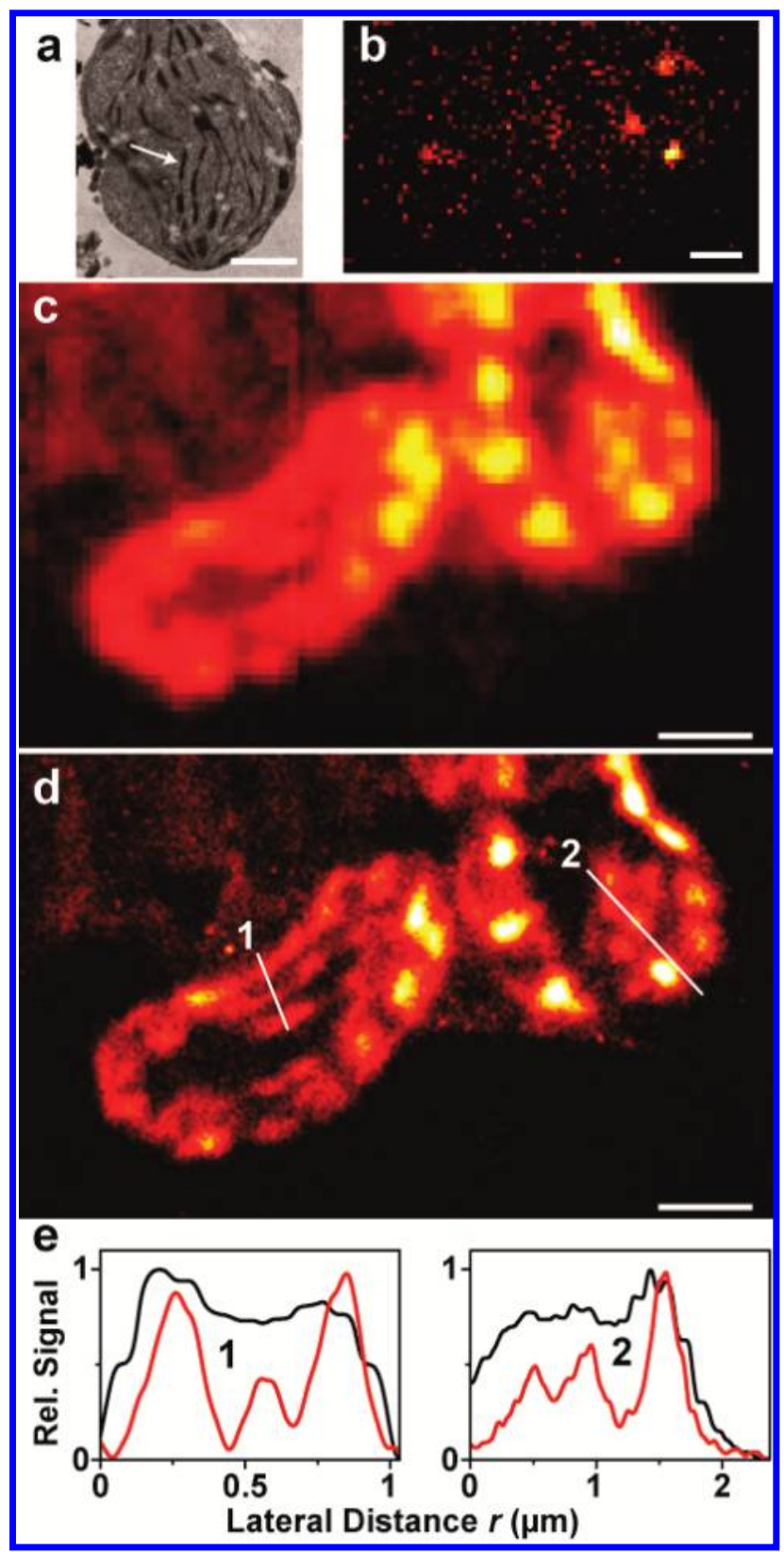

FIGURE 2. Subdiffraction resolution imaging of autofluorescent chloroplasts. (a) Electron micrograph pinpointing (arrow) the thylakoid membranes (darker areas) in the chloroplasts. (b) Typical single camera frame image showing single chlorophyll molecules. (c, d) Autofluorescence wide-field (c) and GSDIM (d) images of thylakoid membranes in the chloroplasts and selected (white line) line profiles (e). The wide-field images were created by summing up all camera frame images after background subtraction. Optical images recorded with a $488 \mathrm{~nm}$ wavelength continuous wave beam of $28 \mathrm{~kW} / \mathrm{cm}^{2}$ focal intensity. Scale bar $1 \mu \mathrm{m}$.

In the following, 174000 camera frames of on-off blinking fluorophores were taken with an exposure time of 10 $\mathrm{ms}$. About $10^{6}$ single-molecule events were registered in total, and a high-resolution GSDIM image reconstructed from their molecular positions (Figure 2d). The thylakoid membrane stack can be clearly observed as well as narrow connections between different stacks. Under the conditions applied, the average number of photons detected per single molecule was about 90 , resulting in a localization accuracy of $\sim 35 \mathrm{~nm} .{ }^{30}$ The actual spatial resolution may have been slightly lower (>40 nm) due to experimental imperfections such as sample drift during the 30 min recording time; nonetheless the level of detail in the GSDIM imaging surpasses by far that of a conventional wide-field image (Figure 2c,e). Similar results were obtained from GSDIM images of other chloroplast samples (Figure S1), even when using 655 $\mathrm{nm}$ for excitation (Figure S2).

Despite the rather long illumination period before starting the measurement (during which quite a substantial number of the chlorophyll molecules turn dark or become photobleached), we can safely assume that the localization of only a subset of chlorophyll molecules was adequate to produce a faithful subdiffraction resolution image of the chloroplast (compare Figure S1). On the other hand, shortening of the preillumination period should allow the recording of less densely packed samples. Unlike in a photoactivation scheme where the density of active fluorophores per diffraction volume can be regulated by the activation light, in GSDIM the regulation of the density of bright molecules has to occur via prebleaching or via a deliberate engineering of the dark state lifetime. ${ }^{22-24,27}$ Here we used the chloroplast slices without further preparation.

In basically all current nanoscopy methods, the resolution depends on the transition (cycling) rates to and from the dark states under the chosen illumination intensity. ${ }^{15,16,19,22}$ In a stochastic method such as GSDIM, the population of the transient dark state has to be large enough to enable just about a single bright state molecule within the diffraction zone. Clearly, this ratio also depends on the local concentration of autofluorescent molecules. On the molecular level, this ratio is optimized by a long dark state lifetime and/or a large probability of crossing to the dark states. Unfortunately, a too long lifetime or a too large crossing probability to dark states result in a too sparse number of on state molecules (and thus a long image acquisition time) or a too low number of emitted photons (i.e., a low localization precision), respectively.

The average dark state population of our chlorophyll samples was $\sim 70 \%$ (Figure 1) and thus lower than the $>90-95 \%$ populations experienced for conventional organic dyes in previous GSDIM experiments. ${ }^{22}$ This seemingly incomplete fluorophore off-turning most likely stems from other autofluorescence background, such as from $\beta$-carotene, or from other not entirely dark chlorophyll states, or be due to the complex set of state transitions in chlorophyll. ${ }^{28,29}$ We note that the population of the dark states may be controlled by additional illumination with another wavelength, ${ }^{22}$ and of course also by specific embedding media, ${ }^{22}$ by additives ${ }^{23,24,27}$ or by any other means that changes the balance between the on-off and the off-on transition rates. In fact, we observed that near-UV illumination increased the return of the chlorophyll fluorescence and thus accelerated the imaging process. 
This acceleration is specifically useful at later camera frames where, due to photobleaching and transitions to long-lived dark states, the density of on molecules becomes very sparse.

It will be interesting to investigate which other autofluorophores and autofluorescent samples provide the right conditions in terms of dark state lifetimes and molecular density to apply GSDIM. Here, we have used neither additional UV illumination nor a specific embedding medium nor added reducing and oxidizing agents nor measured at cryogenic temperatures as in previous GSDIM or GSD recordings applying conventional fluorescence markers. ${ }^{22-24,27}$ Followed by a final washing step only, the cryosliced chloroplast samples were directly employed on the microscope and measured at room temperature (Supporting Information). Hence, one can imagine a number of improvements of the recording strategy, including variations in irradiation intensity and wavelength to further perfect the recording and increase the applicability range. In any case, in the light that all far-field optical nanoscopy approaches have so far relied on fluorescently modified samples, we have now demonstrated that this rapidly emerging field can be extended to the investigation of specimens with molecular constituents left unaltered.

Acknowledgment. We thank Daniel Neumann for help with the samples and Jay Jethwa for a critical reading of the manuscript. This work was supported by the Gottfried Wilhelm Leibniz Program of the Deutsche Forschungsgemeinschaft (to S.W.H.).

Supporting Information Available. Detailed experimental methods for isolation and cryo-sectioning of the chloroplasts and Figures S1 and S2 displaying further GSDIM images of chloroplasts following excitation at 488 and 655 $\mathrm{nm}$, respectively. This material is available free of charge via the Internet at http://pubs.acs.org.

\section{REFERENCES AND NOTES}

(1) Billinton, N.; Knight, A. W. Anal. Biochem. 2001, 291 (2), $175-$ 197.

(2) Min, W.; Lu, S.; Chong, S.; Roy, R.; Holtom, G. R.; Xie, X. S. Nature 2009, 461, 1105-1109.

(3) Hell, S. W.; Wichmann, J. Opt. Lett. 1994, 19 (11), 780-782.

(4) Hell, S. W.; Kroug, M. Appl. Phys. B: Lasers Opt. 1995, 60, 495497.

(5) Klar, T. A.; Jakobs, S.; Dyba, M.; Egner, A.; Hell, S. W. Proc. Natl. Acad. Sci.U.S.A. 2000, 97, 8206-8210.
(6) Heintzmann, R.; Jovin, T. M.; Cremer, C. L. Opt. Soc. Am. A 2002, 19 (8), 1599-1609.

(7) Hell, S. W.; Jakobs, S.; Kastrup, L. Appl. Phvs. A: Mater. Sci. Process. 2003, 77, 859-860.

(8) Hofmann, M.; Eggeling, C.; Jakobs, S.; Hell, S. W. Proc. Natl. Acad. Sci. U.S.A. 2005, 102 (49), 17565-17569.

(9) Gustafsson, M. G. L. Proc. Natl. Acad. Sci.U.S.A. 2005, 102 (37), 13081-13086.

(10) Bretschneider, S.; Eggeling, C.; Hell, S. W. Phvs. Rev. Lett. 2007, 98 (21), 218103.

(11) Betzig, E.; Patterson, G. H.; Sougrat, R.; Lindwasser, O. W.; Olenych, S.; Bonifacino, J. S.; Davidson, M. W.; LippincottSchwartz, J.; Hess, H. F. Science 2006, 313 (5793), 1642-1645.

(12) Rust, M. J.; Bates, M.; Zhuang, X. W. Nat. Methods 2006, 3, 793795.

(13) Hess, S. T.; Girirajan, T. P. K.; Mason, M. D. Biophvs. I. 2006, 91 (11), 4258-4272.

(14) Egner, A.; Geisler, C.; von Middendorff, C.; Bock, H.; Wenzel, D.; Medda, R.; Andresen, M.; Stiel, A.-C.; Jakobs, S.; Eggeling, C.; Schoenle, A.; Hell, S. W. Biophvs. I. 2007, 93, 3285-3290.

(15) Hell, S. W. Science 2007, 316 (5828), 1153-1158.

(16) Hell, S. W. Nat. Methods 2009, 6 (1), 24-32.

(17) Heisenberg, W. The physical principles of the quantum theory; Chicago University Press: Chicago, IL, 1930.

(18) Bobroff, N. Rev. Sci. Instrum. 1986, 57 (6), 1152-1157.

(19) Hell, S. W. Nat. Biotechnol. 2003, 21 (11), 1347-1355.

(20) Tsien, R. Y.; Waggoner, A. Fluorophores for confocal microscopy. In Handbook of Biological Confocal Microscopy; Pawley, J., Ed. Plenum Press: New York, 1995, pp 267-279.

(21) Eggeling, C.; Widengren, J.; Rigler, R.; Seidel, C. A. M. Photostabilities of fluorescent dyes for single-molecule spectroscopy: Mechanisms and experimental methods for estimating photobleaching in aqueous solution. In Applied fluorescence in chemistry, biology and medicine; Rettig, W., Strehmel, B., Schrader, M., Seifert, H., Eds.; Springer: Berlin, 1999; pp 193-240.

(22) Fölling, J.; Bossi, M.; Bock, H.; Medda, R.; Wurm, C. A.; Hein, B.; Jakobs, S.; Eggeling, C.; Hell, S. W. Nat. Methods 2008, 5, $943-$ 945.

(23) Steinhauer, C.; Forthmann, C.; Vogelsang, J.; Tinnefeld, P. L.Am. Chem. Soc. 2008, 130, 16840-16841.

(24) Heilemann, M.; van de Linde, S.; Schuttpelz, M.; Kasper, R.; Seefeldt, B.; Mukherjee, A.; Tinnefeld, P.; Sauer, M. Anqew. Chem. Int. Ed. 2008, 47, 6172-6176.

(25) Biteen, J. S.; Thompson, M. A.; Tselentis, N. K.; Bowman, G. R.; Shapiro, L.; Moerner, W. E. Nat. Methods 2008, 5, 947-949.

(26) Lemmer, P.; Gunkel, M.; Baddeley, D.; Kaufmann, R.; Urich, A.; Weiland, Y.; Reymann, J.; Muller, P.; Hausmann, M.; Cremer, C. Appl. Phvs. B: Lasers Opt. 2008, 93, 1-12.

(27) Vogelsang, J.; Cordes, T.; Forthmann, C.; Steinhauer, C.; Tinnefeld, P. Proc. Natl. Acad. Sci. U.S.A. 2009, 106 (20), 8107-8112.

(28) Porter, G.; Strauss, G. Proc. R. Soc.London, Ser. A 1966, 295, 1 12.

(29) Shuvalov, V. A. Biochim. Biophvs. Acta 1976, 430 (1), 113-121.

(30) Bossi, M.; Fölling, J.; Belov, V. N.; Boyarskiy, V. P.; Medda, R.; Egner, A.; Eggeling, C.; Schoenle, A.; Hell, S. W. Nano Lett. 2008, 8 (8), 2463-2468. 